(6) OPEN ACCESS

\title{
Younger age of onset in familial amyotrophic lateral sclerosis is a result of pathogenic gene variants, rather than ascertainment bias
}

\author{
Puja R Mehta, ${ }^{1,2}$ Ashley R Jones, ${ }^{1}$ Sarah Opie-Martin, ${ }^{1}$ Aleksey Shatunov, ${ }^{1}$ \\ Alfredo lacoangeli, ${ }^{1,3}$ Ahmad Al Khleifat, ${ }^{1}$ Bradley N Smith, ${ }^{1}$ Simon Topp, ${ }^{1}$ \\ Karen E Morrison, ${ }^{4}$ Pamela J Shaw ${ }_{1}^{5}$ Christopher E Shaw, ${ }_{1}^{2,6}$ Sarah Morgan, ${ }^{7}$ \\ Alan Pittman, ${ }^{7}$ Ammar Al-Chalabi ${ }^{1,2}$
}

For numbered affiliations see end of article.

\section{Correspondence to}

Professor Ammar Al-Chalabi, Department of Basic and

Clinical Neuroscience, Maurice Wohl Clinical Neuroscience Institute, King's College London, London SE5 9RX, UK; ammar. al-chalabi@kcl.ac.uk

Received 23 June 2018 Revised 31 July 2018 Accepted 18 August 2018 Published Online First 30 September 2018

\section{Linked}

- http://dx.doi.org/10.1136/ jnnp-2018-319473

Check for updates

(C) Author(s) (or their employer(s)) 2019. Re-use permitted under CC BY. Published by BMJ.

To cite: Mehta PR, Jones AR, Opie-Martin S, et al. J Neurol Neurosurg Psychiatry 2019:90:268-271.

\section{ABSTRACT}

Objective Amyotrophic lateral sclerosis (ALS) is a rapidly progressive neurodegenerative disease of motor neurons with a median survival of 2 years. Familial ALS has a younger age of onset than apparently sporadic ALS. We sought to determine whether this younger age of onset is a result of ascertainment bias or has a genetic basis.

Methods Samples from people with ALS were sequenced for $13 \mathrm{ALS}$ genes. To determine the effect of genetic variation, age of onset was compared in people with sporadic ALS carrying a pathogenic gene variant and those who do not; to determine the effect of family history, we compared those with genetic sporadic ALS and familial ALS.

Results There were 941 people with a diagnosis of ALS, 100 with familial ALS. Of 841 with apparently sporadic ALS, 95 carried a pathogenic gene variant. The mean age of onset in familial ALS was 5.3 years younger than for apparently sporadic ALS ( $p=6.0 \times 10^{-5}, 95 \% \mathrm{Cl} 2.8$ to 7.8 years). The mean age of onset of genetic sporadic ALS was 2.9 years younger than non-genetic sporadic ALS ( $p=0.011,95 \% \mathrm{Cl} 0.7$ to 5.2 years). There was no difference between the mean age of onset in genetic sporadic ALS and familial ALS ( $p=0.097)$.

Conclusions People with familial ALS have an age of onset about 5 years younger than those with apparently sporadic ALS, and we have shown that this is a result of Mendelian gene variants lowering the age of onset, rather than ascertainment bias.

\section{INTRODUCTION}

Amyotrophic lateral sclerosis (ALS), also known as motor neuron disease, is a rapidly progressive and paralysing illness resulting from degeneration of upper and lower motor neurons; it has no cure and the median survival is 2 years. ${ }^{1}$ The mean age of onset in clinic populations is about 58 years, ${ }^{2}$ and in population studies about $64,{ }^{3}$ but it can affect people of any age.

The age of onset of people with familial ALS is widely reported as being about 10 years younger than for those with apparently sporadic ALS. ${ }^{4-6}$ This may be a result of the greater awareness of those who have already experienced ALS in their family, leading them to seek medical attention sooner, and the greater likelihood of recognition of the diagnosis by medical professionals when there is an existing family history. Such ascertainment bias has been proposed as the mechanism for the genetic phenomenon of anticipation, in which genetic diseases seem to occur at younger ages in succeeding generations. ${ }^{7}$ Another explanation, however, is that large-effect Mendelian ALS genes themselves lower the disease age of onset. Studies aiming to investigate genetic factors that influence ALS phenotypes, including age of onset, have yet to yield replicable positive results. ${ }^{8}$

Familial ALS has been described since the mid-1800s, and the rate is reported as $5 \%,{ }^{9}$ $10 \%{ }^{10}$ or as high as $20 \% .^{11}$ The reasons for the discrepancy probably relate, at least in part, to differing personal definitions of a positive family history. ${ }^{12}$ People with ALS but without a family history are described as having sporadic or isolated ALS. Sporadic ALS is sometimes conflated with non-genetic ALS, but familial ALS may be missed, for example, because of the uncertainty around what should constitute a relevant family history, and apparently sporadic ALS may sometimes result from large-effect, rare, Mendelian gene variants. ${ }^{13-15}$ The result is that there are three genetic groups of people with ALS: those with a family history of disease (group 1), those with no family history but a known genetic cause (group 2), and those with no family history and no known genetic cause (group 3). The relative age of onset would be expected to differ between these groups depending on whether ascertainment bias or genetic effects are driving the earlier onset. If ascertainment bias is the explanation for a younger age of onset in familial ALS, group 1 should have a younger age of onset than group 2 , because the only difference is knowledge of family history (both share a genetic basis), whereas if genetic causes are the explanation, group 2 should have a younger age of onset than group 3 , because the only difference is a genetic basis of the disease (in both there is no known family history).

We therefore sought to determine whether the younger age of onset observed in those with familial ALS is a result of ascertainment bias. 


\section{METHODS}

\section{Data sources}

Patients of European ancestry who had contributed to the UK National DNA Bank for Motor Neuron Disease Research were analysed. Age of onset and family history were obtained for each person.

\section{Determination of pathogenic ALS gene variants}

A panel of 13 ALS genes was tested (ANG, C9orf72, DAO, DCTN1, FUS, OPTN, PFN1, SOD1, SQSTM1, TARDBP, $U B Q L N 2, V A P B$ and $V C P$ ), selected for harbouring large-effect, rare, Mendelian ALS gene variants. ${ }^{16} 17$ Pathogenicity was defined based on previously reported association with ALS, or detection in cases, but not in controls or several control databases. ${ }^{17}$ Because of the rapid rate of advance of ALS genetics, more recently identified genes, such as TBK1, were not studied. ${ }^{18}$ DNA was sequenced using Illumina MiSeq technology by means of the Illumina TruSeq Custom Amplicon Assay, as previously described, ${ }^{17}$ and repeat primed PCR used to assay the hexanucleotide repeat expansion in the C9orf72 gene. ${ }^{19}$

Patients were classified into three groups: group 1, familial ALS; group 2, apparently sporadic ALS with a genetic basis; group 3, apparently sporadic ALS without an identified genetic basis.

\section{Statistical analysis}

The mean age of onset between groups was compared with a $t$-test. Uncorrected $\mathrm{p}$ values are reported. To estimate the size of any ascertainment bias observed, the median time between symptom onset and diagnosis was compared between those with familial ALS and those with apparently sporadic ALS, in a MannWhitney U test. Analyses were performed in SPSS V.24.0.

\section{RESULTS}

There were 941 people with ALS included in the study, 841 with apparently sporadic ALS and 100 with familial ALS

\begin{tabular}{|c|c|c|}
\hline Gene & Variant & Cases (n) \\
\hline C9orf72 & Expansion mutation & 34 \\
\hline$D A O$ & p.R199W & 1 \\
\hline \multirow[t]{2}{*}{ FUS } & p.R521H & 2 \\
\hline & p.R514G & 1 \\
\hline \multirow[t]{7}{*}{ SOD1 } & p.C112Y & 1 \\
\hline & p.D91A & 1 \\
\hline & p.L107F & 1 \\
\hline & p.D102N & 1 \\
\hline & p.D102G & 1 \\
\hline & p.1114T & 2 \\
\hline & p.D77Y & 2 \\
\hline SQSTM1 & p.K238E & 2 \\
\hline \multirow[t]{5}{*}{ TARDBP } & p.N378D & 2 \\
\hline & p.A90V & 1 \\
\hline & p.M337V & 1 \\
\hline & p.G348V & 1 \\
\hline & p.Y374X & 1 \\
\hline UBQLN2 & p.P497H & 1 \\
\hline No mutation identified & & 44 \\
\hline Total & & 100 \\
\hline
\end{tabular}

95 of the 100 familial ALS cases were tested for the C9orf72 expansion mutation. (table 1). There were 95 people with apparently sporadic ALS with a presumed pathogenic ALS gene variant (table 2) and 746 without.

The mean age of onset for those with familial ALS was 56.2 years (SD 12.1), all apparently sporadic ALS 61.5 years (SD 11.0), genetically determined apparently sporadic ALS 58.9 years (SD 10.3) and apparently sporadic ALS without a genetic basis 61.8 years (SD 11.1).

The mean age of onset in familial ALS was 5.3 years younger than for apparently sporadic ALS $\left(p=6.0 \times 10^{-5}, 95 \%\right.$ CI for the difference 2.8 to 7.8 years), confirming previous observations that familial ALS has a younger age of onset.

There was no difference between the mean age of onset of people with familial ALS (group 1) and those with genetic apparently sporadic ALS (group 2; $\mathrm{p}=0.097$ ), suggesting that ascertainment bias plays little role in the observed younger age of onset. The mean age of onset of those with genetic apparently sporadic ALS (group 2) was 2.9 years younger than those with non-genetic sporadic ALS (group 3; $\mathrm{p}=0.011$, 95\% CI for the difference 0.7 to 5.2 years), consistent with the hypothesis that genetic burden lowers the age of onset. Combining the genetic groups and comparing them with the non-genetic ALS group confirmed the finding, with a mean age of onset of 3.4 years younger for the genetic groups than the non-genetic group $\left(\mathrm{p}=2.0 \times 10^{-6}, 95 \% \mathrm{CI}\right.$ for the difference 2.5 to 6.1$)$.

The median time between symptom onset and diagnosis was 3 months shorter for familial ALS than apparently sporadic ALS $(\mathrm{p}=0.012)$.

\section{DISCUSSION}

We have confirmed that people with familial ALS have an age of onset about 5 years younger than those with apparently sporadic ALS, as opposed to the widely quoted 10 years, and we propose that this up-to-date figure obtained from a large data set should be used in the clinical setting, particularly when communicating with patients. We have shown that this younger age of onset in familial ALS is a result of Mendelian gene variants lowering the actual age of onset, rather than ascertainment bias resulting in quicker recognition of symptoms. Ascertainment bias does exist, in that people with a family history of ALS are diagnosed on average 3 months more quickly than those without, but the ascertainment bias has no effect on age of onset.

Our findings are consistent with recent findings in which the multistep model of ALS $^{20}$ was applied to three known genetic causes of ALS: mutation in SOD1, mutation in TARDBP (coding for TDP43) and pathological expansion of a hexanucleotide repeat in the C9orf72 gene. ${ }^{21}$ These gene variants account for more than one step in the pathological process leading to ALS, leaving fewer risk factors to encounter before onset and therefore making a younger age of onset more likely. One might also expect some genes or gene variants to show a characteristic spectrum of age of onset, which will become apparent as our genetic knowledge of ALS increases. A similar situation may also exist in other neurodegenerative diseases in which those with a family history have a younger onset than those without, such as Alzheimer's disease and Parkinson's disease.

A weakness of this study is that we only ascertained a genetic basis for ALS using a panel of 13 genes. Even with this restricted set, however, we were able to show that the reduced age of onset is a result of Mendelian disease genes. Furthermore, it is only now, with the availability of high-throughput 
Table 2 Mutations identified in those with apparently sporadic amyotrophic lateral sclerosis

\begin{tabular}{|c|c|c|}
\hline Gene & Variant & Cases (n) \\
\hline \multirow[t]{2}{*}{$A N G$} & p.K78E* & 2 \\
\hline & p.R146H & 1 \\
\hline C9orf72 & Expansion mutation* & 36 \\
\hline$D A O$ & p.R199Q & 1 \\
\hline \multirow[t]{3}{*}{ DCTN1 } & p.V1081M & 1 \\
\hline & p.T12A & 1 \\
\hline & p.R785W & 1 \\
\hline \multirow[t]{6}{*}{ FUS } & p.R269W & 1 \\
\hline & p.R521C & 1 \\
\hline & p.P431L & 1 \\
\hline & p.R521L & 1 \\
\hline & p.G507D & 1 \\
\hline & p.221_221del & 1 \\
\hline \multirow[t]{11}{*}{ OPTN } & p.R545Q & 1 \\
\hline & Exon $7 /$ Intron 7 splice site & 1 \\
\hline & p.Q441X & 1 \\
\hline & p.E380fs & 1 \\
\hline & p.V295F & 1 \\
\hline & p.N303K & 1 \\
\hline & p.G509R & 1 \\
\hline & p.A184V* & 1 \\
\hline & p.K413fs* & 1 \\
\hline & p.R271H* & 1 \\
\hline & p.F226S & 1 \\
\hline \multirow[t]{2}{*}{ PFN1 } & p.E117D* & 1 \\
\hline & p.E117G* & 1 \\
\hline \multirow[t]{5}{*}{ SOD1 } & p.D91A & 1 \\
\hline & p.T40A* & 1 \\
\hline & p.S135G* & 1 \\
\hline & p.I114T & 2 \\
\hline & p.L9V & 1 \\
\hline \multirow[t]{2}{*}{ SQSTM1 } & p.K238E* & 7 \\
\hline & p.P392L* & 2 \\
\hline \multirow[t]{2}{*}{ TARDBP } & p.G2875* & 3 \\
\hline & p.A90V & 1 \\
\hline \multirow[t]{5}{*}{ UBQLN2 } & p.A603D & 1 \\
\hline & р.Т334M & 3 \\
\hline & p.L87F & 1 \\
\hline & p.P497H & 1 \\
\hline & p.496_499del & 1 \\
\hline \multirow[t]{3}{*}{$V A P B$} & p.158_159del* & 7 \\
\hline & p.M170"* & 5 \\
\hline & p.R184Q & 1 \\
\hline \multirow[t]{2}{*}{$V C P$} & p.I114V & 1 \\
\hline & p.G523V & 1 \\
\hline Total & & $\begin{array}{l}103 \text { variants } \\
\text { (95 people) }\end{array}$ \\
\hline
\end{tabular}

In some cases, an individual had more than one pathogenic gene variant; such cases are indicated by an asterisk and counted for each variant. Three of these had C9orf72 expansion as one of the variants.

gene sequencing, that a study like this is possible, since the prospect of sequencing a panel of 13 genes in more than 900 people would not have been feasible a few years ago.

Genetic modulators of the age of ALS onset have been studied before, ${ }^{82-27}$ but not replicated. The explanation for why these have not been replicated may be that genetic risk factors themselves lower the age of onset and other genetic variants that do not influence risk have only a limited effect on age of onset, making them difficult to detect, even with large sample sizes.

Although we have identified this genetic effect on age of onset using large-effect, rare variants, the liability threshold model of disease ${ }^{2829}$ would suggest that all genetic contributors to ALS risk lower age of onset, even common variants that have a small effect on risk. In the liability threshold model, there is a normal distribution of the burden of genetic and environmental risk factors (liabilities) across the population. Most individuals who are unaffected will possess some degree of liability; however, only individuals with a burden of risk factors sufficient to cross a threshold develop a disease. A consequence of the model is that affected individuals with the least at-risk phenotype are also those with a higher liability than average, since the threshold must be higher for a low-risk phenotype (that is why it is low risk), and yet they still have sufficient burden to cross it. One of the strongest risk factors for ALS is increased age, and therefore younger patients must carry a higher burden of risk factors. Thus, genetic burden is likely to be higher in younger patients, and conversely the age of onset will be lower in those with a higher genetic burden. One study has shown that people with ALS harbouring variants in more than one gene had a disease onset 10 years earlier than those with variants identified in only one gene, supporting the idea that genetic burden influences age of onset. ${ }^{30}$

Our findings show that Mendelian gene variants are associated with a younger age of onset in ALS, regardless of family history. Further studies are needed to elucidate the mechanisms by which this occurs.

\section{Author affiliations}

${ }^{1}$ Department of Basic and Clinical Neuroscience, Maurice Wohl Clinical Neuroscience Institute, King's College London, London, UK

${ }^{2}$ Department of Neurology, King's College Hospital, Denmark Hill, London, UK ${ }^{3}$ Department of Biostatistics and Health Informatics, Institute of Psychiatry,

Psychology and Neuroscience, King's College London, London, UK

${ }^{4}$ Faculty of Medicine, University of Southampton, University Hospital Southampton NHS Foundation Trust, Southampton, UK

${ }^{5}$ Sheffield Institute for Translational Neuroscience (SITraN), University of Sheffield Sheffield, UK

${ }^{6}$ Institute of Psychiatry, Psychology and Neuroscience, UK Dementia Research Institute, Maurice Wohl Clinical Neuroscience Institute, King's College London, London, UK ${ }^{7}$ Department of Molecular Neuroscience, Institute of Neurology, UCL, Queen Square, London, UK

Acknowledgements The samples used in this research were obtained from the UK National DNA Bank for MND Research, funded by the MND Association and the Wellcome Trust. We acknowledge sample management undertaken by Biobanking Solutions funded by the Medical Research Council at the Centre for Integrated Genomic Medical Research, University of Manchester.

Contributors PRM and AA-C: conception and design, analysis, and interpretation of data. PRM, ARJ, SO-M, AS, BS, Al, ST, PJS, KEM, CES and AA-C: acquisition of data. All authors: drafting and revising the article. PRM, SM, AP and AA-C: revising the article critically for important intellectual content.

Funding This is an EU Joint Programme - Neurodegenerative Disease Research (JPND) project. The project is supported through the following funding organisations under the aegis of JPND (www.jpnd.eu): UK Medical Research Council (MR/ L501529/1 STRENGTH; MR/R024804/1 BRAIN-MEND) and the Economic and Social Research Council (ES/L008238/1 ALS-CarE) and through the Motor Neurone Disease Association. This study represents independent research part-funded by the National Institute for Health Research (NIHR) Biomedical Research Centre at South London and Maudsley NHS Foundation Trust and King's College London. The work leading up to this publication was funded by Horizon 2020 Programme (H2020-PHC-2014two-stage; grant agreement number 633413). PRM is supported by an NIHR Medical Academic Clinical Fellowship. PJS is supported by an NIHR Senior Investigator award (NF-SI-0512-10082) and the NIHR Sheffield Biomedical Research Centre (Translational Neuroscience IS-BRC-1215-20017). 
Competing interests $\mathrm{AA}-\mathrm{C}$ reports consultancies from Mitsubishi Tanabe Pharma, Chronos Therapeutics, Orion Pharma and Cytokinetics. AA-C was chief trial investigator for Orion Pharma (NCT02487407) and Cytokinetics (NCT02496767). Al other authors declare no conflicts of interest.

\section{Patient consent Obtained.}

Ethics approval Patients were analysed under ethical approval reference 08/ H0405/60 from the Trent Research Ethics Committee.

Provenance and peer review Not commissioned; externally peer reviewed.

Data sharing statement Data are available for download by application to the MND Association.

Open access This is an open access article distributed in accordance with the Creative Commons Attribution 4.0 Unported (CC BY 4.0) license, which permits others to copy, redistribute, remix, transform and build upon this work for any purpose, provided the original work is properly cited, a link to the licence is given, and indication of whether changes were made. See: http://creativecommons.org/ licenses/by/4.0/

\section{REFERENCES}

1 van Es MA, Hardiman O, Chio A, et al. Amyotrophic lateral sclerosis. Lancet 2017;390:2084-98.

2 Huisman MH, de Jong SW, van Doormaal PT, et al. Population based epidemiology of amyotrophic lateral sclerosis using capture-recapture methodology. J Neurol Neurosurg Psychiatry 2011;82:1165-70.

3 Marin B, Logroscino G, Boumédiene F, et al. Clinical and demographic factors and outcome of amyotrophic lateral sclerosis in relation to population ancestral origin. Eur J Epidemiol 2016;31:229-45.

4 Kurland LT, Choi NW, Sayre GP. Implications of incidence and geographic patterns on the classification of amyotrophic lateral sclerosis. In: Norris FH, ed. Motor neuron diseases: research on amyotrophic lateral sclerosis and related disorders. New York and London: Grune and Stratton, 1968: 28-50.

5 Li TM, Alberman E, Swash M. Comparison of sporadic and familial disease amongst 580 cases of motor neuron disease. J Neurol Neurosurg Psychiatry 1988:51:778-84.

6 ALS research collaboration. 2018.About familial als university of miami https://www. als-research.org/familial-als/\#toggle-id-6 (accessed 1 Jun 2018).

7 Penrose LS. The problem of anticipation in pedigrees of dystrophia myotonica. Ann Eugen 1948; 14:125-32.

8 Ahmeti KB, Ajroud-Driss S, Al-Chalabi A, et al. Age of onset of amyotrophic lateral sclerosis is modulated by a locus on 1p34.1. Neurobiol Aging 2013;34:357.e7-19.

9 Byrne S, Walsh C, Lynch C, et al. Rate of familial amyotrophic lateral sclerosis: a systematic review and meta-analysis. J Neurol Neurosurg Psychiatry 2011;82:623-7.

10 Kurland LT, Mulder DW. Epidemiologic investigations of amyotrophic lateral sclerosis. 2. Familial aggregations indicative of dominant inheritance. I. Neurology 1955;5:182-96.
11 Andersen PM, Al-Chalabi A. Clinical genetics of amyotrophic lateral sclerosis: what do we really know? Nat Rev Neurol 2011;7:603-15.

12 Byrne S, Elamin M, Bede P, et al. Absence of consensus in diagnostic criteria for familial neurodegenerative diseases. J Neurol Neurosurg Psychiatry 2012;83:365-7.

13 Al-Chalabi A, Lewis CM. Modelling the effects of penetrance and family size on rates of sporadic and familial disease. Hum Hered 2011;71:281-8.

14 van Rheenen W, Shatunov A, Dekker AM, et al. Genome-wide association analyses identify new risk variants and the genetic architecture of amyotrophic lateral sclerosis. Nat Genet 2016;48:1043-8.

15 Al-Chalabi A. Perspective: don't keep it in the family. Nature 2017;550:S112.

16 Brown RH, Al-Chalabi A, Sclerosis AL. Amyotrophic lateral sclerosis. N Engl J Med 2017;377:162-72.

17 Morgan S, Shatunov A, Sproviero W, et al. A comprehensive analysis of rare genetic variation in amyotrophic lateral sclerosis in the UK. Brain 2017;140:1611-8.

18 Cirulli ET, Lasseigne BN, Petrovski S, et al. Exome sequencing in amyotrophic lateral sclerosis identifies risk genes and pathways. Science 2015;347:1436-41.

19 DeJesus-Hernandez M, Mackenzie IR, Boeve BF, et al. Expanded GGGGCC hexanucleotide repeat in noncoding region of C9ORF72 causes chromosome 9p-linked FTD and ALS. Neuron 2011;72:245-56.

20 Al-Chalabi A, Calvo A, Chio A, et al. Analysis of amyotrophic lateral sclerosis as a multistep process: a population-based modelling study. Lancet Neurol 2014;13:1108-13

21 Chio A, Mazzini L, D'Alfonso S, et al. Genetic mutations in the six-steps process in ALS. Neurology. In Press:2018.

22 Gros-Louis F, Andersen PM, Dupre N, et al. Chromogranin B P413L variant as risk factor and modifier of disease onset for amyotrophic lateral sclerosis. Proc Natl Acad Sci U S A 2009; 106:21777-82

23 Mitchell J, Morris A, de Belleroche J. Thioredoxin reductase 1 haplotypes modify familial amyotrophic lateral sclerosis onset. Free Radic Biol Med 2009;46:202-11.

24 Li YJ, Pericak-Vance MA, Haines JL, et al. Apolipoprotein E is associated with age at onset of amyotrophic lateral sclerosis. Neurogenetics 2004;5:209-13.

25 Oosthuyse B, Moons L, Storkebaum E, et al. Deletion of the hypoxia-response element in the vascular endothelial growth factor promoter causes motor neuron degeneration. Nat Genet 2001;28:131-8.

26 Fogh I, Rijsdijk F, Andersen PM, et al. Age at onset in sod1-mediated amyotrophic lateral sclerosis shows familiality. Neurogenetics 2007:8:235-6.

27 Black HA, Leighton DJ, Cleary EM, et al. Genetic epidemiology of motor neuron disease-associated variants in the Scottish population. Neurobiol Aging 2017;51: e20:e11-78.

28 Falconer DS. Threshold characters. In: Introduction to quantitative genetics. New York: The Ronald Press Company, 1960: 301-11.

29 Yang J, Visscher PM, Wray NR. Sporadic cases are the norm for complex disease. European Journal of Human Genetics 2010;18:1039-43.

30 Cady J, Allred P, Bali T, et al. Amyotrophic lateral sclerosis onset is influenced by the burden of rare variants in known amyotrophic lateral sclerosis genes. Ann Neurol 2015;77:100-13. 